\title{
Evaluation of C-reactive protein and haptoglobin as malaria episode markers in an area of high transmission in Africa
}

\author{
N. Hurt ${ }^{1}$, T. Smith ${ }^{1,2}$, M. Tanner ${ }^{1,2}$, S. Mwankusye ${ }^{1}$, G. Bordmann ${ }^{2}$, N. A. Weiss ${ }^{2}$ and T. Teuscher ${ }^{1}{ }^{1}$ Ifakara \\ Centre, P.O. Box 53, Ifakara, Tanzania; ${ }^{2}$ Swiss Tropical Institute, P.O. Box, 4002 Basel, Switzerland
}

\begin{abstract}
Field studies of malaria in endemic areas frequently use the presence or levels of parasitaemia, together with the measurement of fever, as the primary criteria with which to identify cases. However, since malaria cases do not always present with measurable fever, and since asymptomatic parasitaemia occurs, additional episode markers might be useful epidemiological tools. We have measured the C-reactive protein and haptoglobin levels in paediatric patients presenting to a village health post in the Kilombero District in Tanzania and in convalescent sera from the same patients, in order to evaluate these acute-phase reactants as alternative markers of Plasmodium falciparum episodes. Among afebrile patients, C-reactive protein levels were highly correlated with parasite density. High C-reactive protein levels are therefore probably indicative of recent clinical malaria cpisodes in currently afebrile individuals with high parasite densities. An appropriate case definition for malaria in epidemiological studies in endemic areas might therefore be hyperparasitaemia accompanied by either, or both, measurable fever and raised C-reactive protein levels. This would give less biased estimates of the overall burden of malaria morbidity than does a definition which requires measurable fever. Levels of haptoglobin were highly negatively correlated with parasitaemia, but did not appear to be useful episode markers because this correlation was probably not related to acute morbidity. However, haptoglobin can be useful to assess at community level the impact of interventions on parasitaemia.
\end{abstract}

\section{Introduction}

A broad spectrum of symptoms is found in clinical malaria in children living in endemic areas. Malaria parasites are often tolerated without development of any obvious signs of disease (GILLES, 1988; GREENWOOD, 1987), and there is no unambiguous diagnostic criterion.

Since fever is the predominant symptom in clinical malaria (GOVARDHINI et al., 1991; STEIN \& GELFAND, 1985), epidemiological studics frequently adopt case dcfinitions which use fever as a non-specific marker to define disease episodes. Parasitaemia is then used as a malaria-specific component to determine which, or what proportion of, the febrile episodes should be classified as malaria (LYIMO et al., 1991; MENON et al., 1990; MNZAva et al., 1993; SNow et al., 1988). Since body temperature fluctuates during acute episodes of malaria (KWIATKOWSKI \& GREENWOOD, 1989), some of the true cases will present afcbrile, leading to under-estimation of the true burden of malaria morbidity. A higher sensitivity might be achievcd by an episode definition based on morc stable markers than fever alone. Parasitacmia could still be used as the malaria-specific component in such a case definition.

Biochemical markers of morbidity such as acule phase proteins might be useful in constructing such case definitions. Such proteins have been used in malaria studies at both the individual and the population level (CHIEWSILP et al., 1988; GILlESPIE et al., 1991; GRANINGER et al., 1992; ROUGEMONT et al., 1988; SISAY et al., 1992). C-reactive protein (CRP) is the classical acute phase reactant and has been advocated as a replacement for the erythrocyte sedimentation rate as a general screening test for disease (FLECK \& MYERS, 1985). CRP levels are raised during acute attacks of Plasmodium falciparum malaria (CHAGNON et al., 1992; NAIK \& Voller, 1984; REE, 1971).

Parasite densities in the peripheral blood are not stable during malaria episodes due to the parasite's life cycle (KWIATKOWSKI et al., 1989) and to antiparasitic regulatory effects in the host (KWIATKOWSKI, 1991). Just as fluctuations in body temperature during episodes lead to underestimation of the prevalence of episodes, fluctuations in parasite densities lead to underestimation of the proportion of episodes associated with high parasitaemia. An acute phase reactant which is indirectly associated with parasite densities is haptoglobin. Malaria episodes are known to be associated with markedly reduced hapAddress for correspondence: Nicole IIurt, Department of Public Health and Epidemiology, Swiss Tropical Institute, P.O. Box, CH-4002 Basel, Switzerland. toglobin levels in non-immunes (GRANINGER et al., 1992). In studies in endemic areas, RougEMONT et al. (1988) and TRAPE et al. (1985) reported that low haptoglobin levels reflect recent parasitaemia and malariainduced haemolysis, whilst other disease conditions result in increased haptoglobin levels.

In this paper we report on an evaluation of the use of serum concentrations of CRP in the definition of malaria episodes in a health facility based study conducted in a rural village in the Kilombero District of south-eastern Tanzania. We also investigated whether haptoglobin might serve as a malaria-specific marker which varies less rapidly than parasite densities.

\section{Materials and Methods}

Study area and study population

The study was undertaken in the Kilombero District, Morogoro Region, Tanzania. The geographic, demographic and health aspects of the study area have been described in detail by TANNER et al. (1987, 1991). Two neighbouring villages lying in the Kilombero river plain are the subject of research activities of the Kilombero Malaria Project ${ }^{\star}$ (KILOMBERO MALARIA PROJECT, 1992; SMITH et al., 1993; TEUSCHER, 1992). The area is highly endemic for $P$. falciparum malaria. Rainfall is highly seasonal, with the heaviest rains occurring from March to May and some occurring in December. The major vectors are Anopheles gambiae sensu lato and $A$. funestus.

Patients were sampled by two of us (N.H., S.M.) during routine consultations at the Namawala Village Health Post (VHP) at which we assisted the village health worker. VHPs are the lowest level health facilities in Tanzania offering mainly basic preventive and curative health services and serving as referral stations (M.O.H., 1983). Patients were selected during a period of 8 weeks in June and July 1991 and again for 4 weeks in October and November 1991. Only patients younger than 15 years and living in Namawala were enrolled in the study. They were initially classified according to the extent of evidence for a febrile condition into one of the following 3 categories. Patients who did not correspond to these categorics werc excluded from the analyses.

(i) Febrile patients were those who were reported, by the child or its guardian, to have had fever both at pres-

\footnotetext{
* The Kilombero Malaria Project is being carried out at the Ifakara Centre (Kilombero District, Tanzania) in collaboration with the Tanzanian National Institute of Medical Research by the Swiss Tropical Institute (Switzerland), Imperial College (England), The Universities of Wageningen and Nijmegen (The Netherlands), and IRTC-WHO Geneva (Switzerland).
} 
ent and also during the previous night, and the axillary temperature on examination of the child was $37.5^{\circ} \mathrm{C}$ or higher. Patients whose febrile illness was reported as beginning on the day of examination were also included in this category.

(ii) Nocturnal fever patients were those in whom a febrile illness was reported as having occurred during the previous night, but with no current fever when they presented at the VHP.

(iii) $\Lambda$ febrilc patients included only those with temperatures below $37.5^{\circ} \mathrm{C}$ and who reported no febrile illness during the previous $48 \mathrm{~h}$.

Whenever possible one age-matched afebrile patient and one nocturnal fever patient were studied together with each febrile patient.

Symptoms and history of fever and antimalarial drug consumption were obtained from the child or its guardian and recorded. Capillary blood was obtained for blood slide examination and for serum collection. Patients were asked to return to the VHP after $24-36 \mathrm{~h}$ and after 7-14 d. Those who did not report were followed up at their home. The same questionnaire was administered, a blood slide taken and a serum sample drawn. Suspected malaria was treated immediately in young children or the following day, based on a malaria-positive blood film. Treatment, based on the Tanzanian essential drugs programme guidelines, was with chloroquine on 3 consecutive days $(10,10$, and $5 \mathrm{mg}$ chloroquine base $/ \mathrm{kg}$ body weight). Non-malarial patients were treated appropriately or referred to other health facilitics.

Community controls, used in the estimation of the sensitivity and specificity of case definitions, were afebrile children under 6 years of age sampled during household surveys between June 1989 and November 1991, as described elsewhere (SMITH et al., 1993; TEUSCHER, 1992).

\section{Laboratory investigations}

Acute phase reactants. Serum concentrations of CRP and haptoglobin were measured using a radial immunodiffusion (RID) assay (The Binding Site, Birmingham, UK). The detection range was $3.3-80.8 \mathrm{mg} / \mathrm{L}$ for CRP and $211-3910 \mathrm{mg} / \mathrm{L}$ for haptoglobin. The immunoprecipitin ring was examined with a Wild M8 stereomicroscope at $12.5 \times$ using a graticule with scale

Parasite densities. Geimsa-stained thick blood films were checked at the VHP for rapid diagnosis and examined again at the laboratory for precise determination of parasitaemia. Parasite densities were calculated by assuming a standard mean leucocyte count of $8000 / \mu \mathrm{L}$ (SHUTE, 1988).

\section{Statistical methods}

Morbidity associated with raised parasitaemia was used as a working definition of a malaria case. Although it is not possible to determine whether the parasitaemia is raised in any individual case, the proportion of patients with raised parasitaemias (the attributable fraction, $\lambda$ ) can be determined by comparing the distribution of parasite densities in cases with that in community controls (Smith, T., Hayes, R., Schellenberg, J. A. \& Teuscher, $T$., paper submitted for publication). So that the same group of controls could be used as in that study, sensitivities and specificities were estimated for patients under 6 years of age only.

In order to estimate the total number of cases with raised parasitaemia, all 3 groups of patients were aggregated (to give a total of $N$ cases). A logistic regression model (Smith ct al., paper submitted for publication) was used to estimate $R_{\mathrm{i}}$, the relative risk of being a case, associated with the parasitaemia found in case $i$, and hence $\lambda_{t}$, the proportion of all the cases with parasitaemia raised above levels in the community:

$$
\lambda_{t}=\frac{1}{N} \sum_{i=1}^{N} \frac{R_{i}-1}{R_{i}}
$$
$N \lambda_{\text {t. }}$

The cstimated total number of malaria cases was then

Each case definition evaluated used 2 cut-offs: a cut-off for CRP, $c$; and a parasitaemia cut-off, $d$. A subset of $n_{c, d}$ cases was identified, which included all measured fever cases with parasitaemia above $d$ together with those nocturnal fever or afebrile cases with CRP level $>c$ and parasitaemia $>d$, Let $\delta_{c, d, i}=1$ where case $i$ is included in this case definition and $\delta_{c, d, i}=0$ otherwise. $\lambda_{c, d}$, the attributable proportion of these cases, was then estimated by including only this reduced set of cases in the summation, i.e.:

$$
\lambda_{c, d}=\frac{1}{n_{c, d}} \sum_{i=1}^{N} \delta_{c, d, i} \frac{R_{i}-1}{R_{i}}
$$

The number of cases correctly identified by the case definition was then estimated as $n_{c, d} \lambda_{c, d}$ and the number of normal individuals incorrectly classified as cases as $n_{c, d}$ $\left(1-\lambda_{c, d}\right)$.

Estimates of the sensitivity and specificity of this case definition are:

$$
\text { Sensitivity }=\frac{n_{c, d} \lambda_{c, d}}{N \lambda_{t}} \text { and specificity: } 1-\frac{n_{c, d}\left(1-\lambda_{c, d}\right)}{N\left(1-\lambda_{\imath}\right)}
$$

\section{Results}

Table 1 shows the mean ages and average levels of CRP and haptoglobin for the 3 groups of VHP patients. The distributions of CRP and haptoglobin levels were very skewed, so medians are presented and non-parametric tests were used to analyse them. The results of nonparametric analyses of variance (Van der Waerden's tests), carried out to test the inter-group differences in CRP and haptoglobin levels, are given in the footnote to Table 1. CRP levels were highest in the fever patients, intermediate in the nocturnal febrile patients, and lowest in the afebrile patients, and differences between all groups were significant. The corresponding test for haptoglobin levels indicated that differences in haptoglobin levels between the 3 groups of patients could easily be

\begin{tabular}{|c|c|c|c|c|c|c|c|c|c|}
\hline \multirow[b]{2}{*}{ Group } & \multicolumn{3}{|c|}{ Age (years) } & \multicolumn{3}{|c|}{ C-reactive protein ${ }^{a}(\mathrm{mg} / \mathrm{L})$} & \multicolumn{3}{|c|}{ Haptoglobin ${ }^{\mathrm{b}}(\mathrm{mg} / \mathrm{L})$} \\
\hline & No. & Mean & $\mathrm{SD}$ & Median & Maximum & $\rho_{1}$ & Median & Maximum & $\rho_{2}$ \\
\hline $\begin{array}{l}\text { Febrile } \\
\text { Afebrile } \\
\text { Nocturnal fever }\end{array}$ & $\begin{array}{l}95 \\
77 \\
69\end{array}$ & $\begin{array}{l}3 \cdot 2 \\
5 \cdot 8 \\
3 \cdot 6\end{array}$ & $\begin{array}{l}3 \cdot 6 \\
4 \cdot 8 \\
3 \cdot 4\end{array}$ & $\begin{array}{r}40 \cdot 7 \\
3 \cdot 3 \\
16 \cdot 3\end{array}$ & $\begin{array}{l}80 \cdot 8^{c} \\
80 \cdot 8^{c} \\
80 \cdot 8^{c}\end{array}$ & $\begin{array}{l}0.06(P=0.57) \\
0.37(P=0.001) \\
0.37(P=0.003)\end{array}$ & $\begin{array}{l}299 \cdot 5 \\
442 \cdot 5 \\
426 \cdot 0\end{array}$ & $\begin{array}{l}3910 \cdot 0^{\mathrm{c}} \\
3170 \cdot 0^{0} \\
3910 \cdot 0^{\mathrm{c}}\end{array}$ & $\begin{array}{l}-0.43(P=0.0001) \\
-0.35(P=0.002) \\
-0.38(P=0.001)\end{array}$ \\
\hline
\end{tabular}
due to chance.

Table 1. C-reactive protein and haptoglubin levels in patients from a malaria endemic area in Tanzania

${ }^{a}$ Van der Waerden's test of significance of difference in C-reactive protein levels between groups: $\chi_{2}^{2}=51 \cdot 0, P<0 \cdot 0001$; $\rho_{1}=$ Spearman partial correlation coefficient between parasite density and C-reactive protein level, controlling for age. bVan der Waerden's test of significance of difference in haptoglobin levels between groups: $\chi_{2}^{2}=0.8, P=0.67$; $\rho_{2}=$ Spearman partial correlation coefficient between parasite density and haptoglobin level, controlling for age.

'Upper limit of concentration recorded by the assay technique. 
Table 2 summarizes the parasite density distributions for the 3 groups of patients on presentation, and for the community controls. The afebrile patients had the lowest parasite densities on average, the febrile patients the highest, and the nocturnal fever patients and community con-

Table 2. Malaria parasite prevalences and densities

\begin{tabular}{lccr}
\hline Group & $\begin{array}{c}\text { No. of } \\
\text { subjects }^{\mathrm{a}}\end{array}$ & $\begin{array}{c}\text { Parasite } \\
\text { prevalence } \\
(\%)\end{array}$ & $\begin{array}{r}\text { Median } \\
\text { parasite } \\
\text { no./ } \mu \mathrm{L}^{\mathrm{b}}\end{array}$ \\
\hline Febrile patients & 73 & $89 \cdot 0$ & 15040 \\
Afebrile patients & 41 & $70 \cdot 7$ & 800 \\
Nocturnal fever patients & 57 & $86 \cdot 0$ & 2400 \\
Community controls & 1859 & $91 \cdot 4$ & 1660
\end{tabular}

${ }^{a}$ Only children under 6 years of age are included.

'Van der Waerden's test of significance of difference in parasite density between groups: $\chi_{3}^{2}=70^{\cdot} 9, P<0 \cdot 0001$.

trols had similar parasite densities. The differences were highly significant.

Table 1 also gives the rank correlations between parasite density and levels of CRP and haptoglobin. There were significant positive correlations between CRP and parasitaemia among the afebrile and the nocturnal fever patients, suggesting that CRP levels are indeed higher in the malaria cases than in the other afebrile cases. Among fever cases there was very little correlation between CRP level and parasite density. In contrast, haptoglobin levels were negatively correlated with parasite density in all 3 groups.

One day after presentation, the median temperatures of all 3 groups of patients were similar and in the normal range. In contrast, parasitc densitics in fcbrile and nocturnal fever patients fell gradually. After one day they were intermediate between those on presentation and those in the community, but by 6-9 d they had fallen to a level well below those of community controls. The low parasitaemias during convalescence were presumably due to the chloroquine therapy, since $77 \%$ of the patients received chloroquine at some stage of study enrolment, including $51 \%$ of initially afebrile patients.

CRP levels initially rose in the patients who presented with fever and then fell to a low (normal) level, which was similar in all 3 groups (Fig. 1). The rank correlation coefficient between initial remperature and CRP level measured one day later was 0.49 , higher than that between initial temperature and temperature measured after one day $(0 \cdot 38)$. Correlations between axillary temperatures measured on different days were consistently positive but were low (Fig. 2). There was a slight tendency for the correlation to decrease when the measurement interval increased. Only for short intervals of $1-2 \mathrm{~d}$ was there a high serial correlation between parasite density measurements. It fell to about 0.1 at an interval duration of $4 \mathrm{~d}$ and levelled at about 0.4 for longer intervals

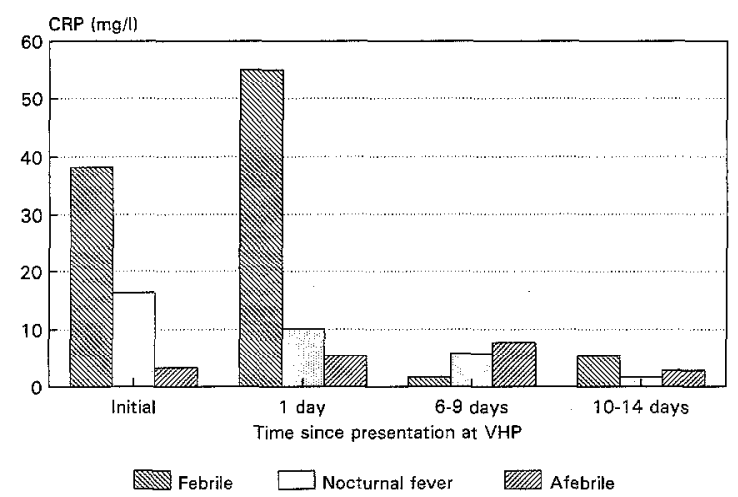

Fig. 1. Changes in median $\mathrm{C}$-reactive protein (CRP) level during followup $(\mathrm{VHP}=$ village heatth post $)$.
Serial Rank Correlation Coefficient

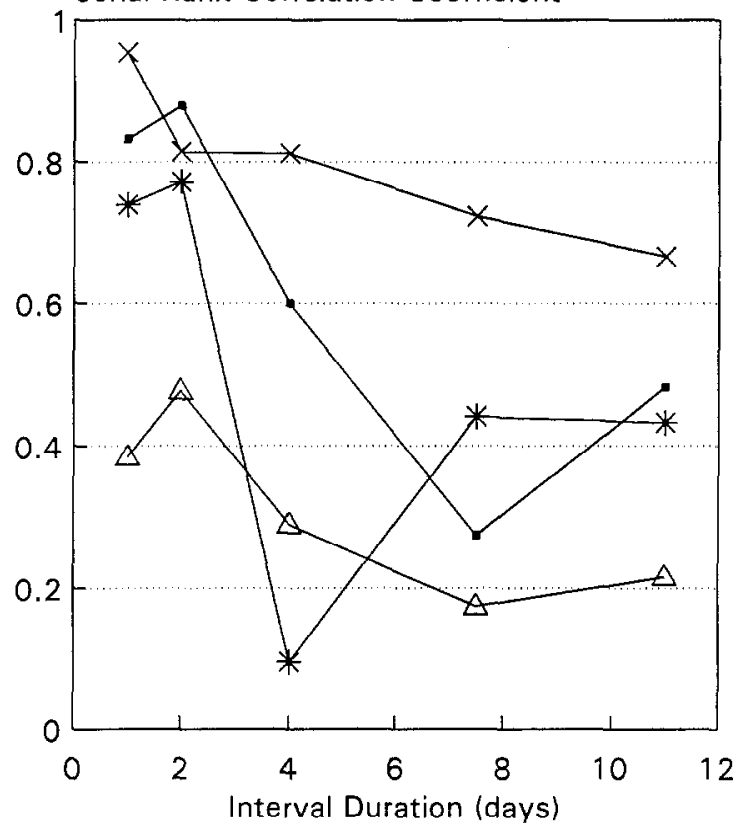

Fig. 2. Serial rank correlations between indicators of morbidity during follow-up of all patients recruited at the village health post, up to $11 \mathrm{~d}$ after first presentation. $\mathbf{E}$, C-reactive protein; $\star$, parasite density; $\times$, haptoglobin; $\Delta$, axillary temperature.

studied. A similar pattern was observed for CRP, although the fall in serial correlation appeared to be rather more gradual than for parasite densities. The low correlation between initial parasite density and that $4 \mathrm{~d}$ after presentation was presumably also due to the chloroquine treatment.

During convalescence, there was no clear pattern of changes in haptoglobin levels. Haptoglobin generally showed the highest serial correlation, with only a gradual falling off to about 0.7 at $11 \mathrm{~d}$. However, the rank correlation between parasite densities on presentation and haptoglobin levels after one day $(-0.38)$ was smaller than that between initial parasitaemia and parasitaemia after one day $(0 \cdot 59)$.

When CRP cut-offs were included in the case definition but no qualifying level of parasitaemia was required, the results illustrated in Fig. 3(a) were obtained. For each level of CRP, $c$, the case definition includes all measured fever cases, together with those nocturnal fever or afebrile cases with CRP level $>c$. The cut-off value of $c=0 \mathrm{mg} / \mathrm{L}$ classifies all the patients as cases, whilst $c=\infty$ classifies only fever patients as cases, ignoring the CRP levels. A reasonably specific case definition (specificity $>60 \%$ ) is obtained only with a high CRP cut-off of at least $c>75 \mathrm{mg} / \mathrm{L}$. Such a definition shows little improvement in sensitivity over the definition with $c=\infty$.

The specificity of the CRP dependent case definitions was increased when the parasite density for cases was required to exceed a threshold $d$ (Fig. 3(b-e)). A value of $d=2000 / \mu \mathrm{L}$ resulted in estimated specificity close to $80 \%$ over most of the range of $c$ values. At the same time, increasing the value of $d$ resulted in a reduction in the sensitivity for any given $c$ value. At high values of $d$ the case definitions showed relatively little dependence on $c$ because cases with very high parasitaemia almost all have high CRP anyway. However, the incorporation of CRP dependence into the definition means that a high specificity can be achieved at a lower value of $d$. For most purposes, a parasitaemia threshold of $d=5000 / \mu \mathrm{L}$ appears to be close to optimal, with a CRP cut-off value between 0 and $40 \mathrm{mg} / \mathrm{L}$, depending upon the relative importance of specificity and sensitivity.

\section{Discussion}

Parasite densities were correlated with CRP concentra- 
(a)

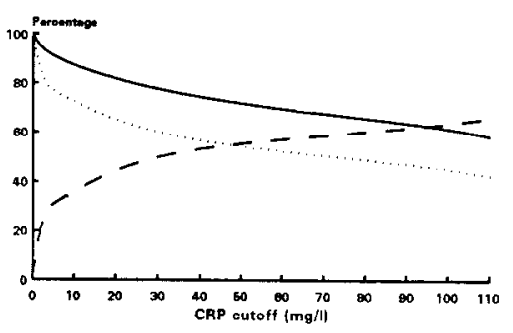

(b)

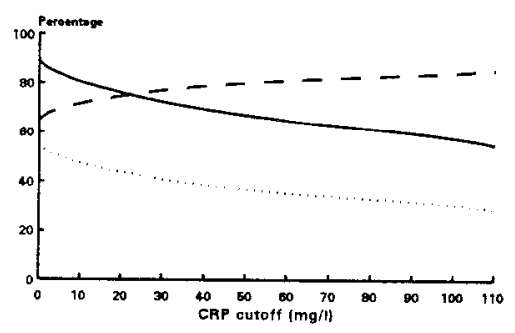

(c)

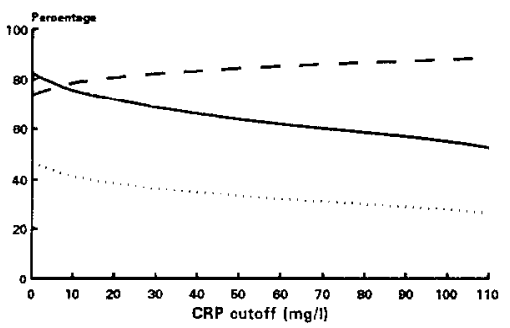

(d)

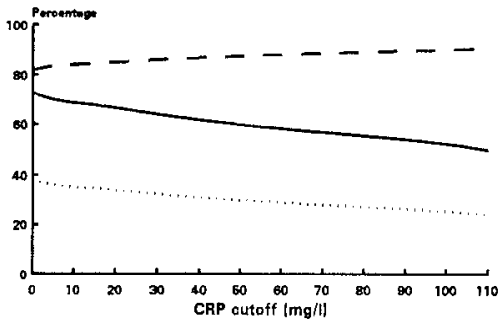

(e)

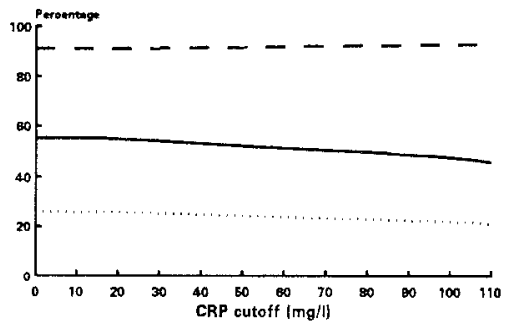

Fig. 3. Proportions of patients classified as malaria cases (....) and estimated sensitivities $(-)$ and specificities ( - - ) for case definitions dependent on the level of ( -reactive protein (CRP) in the blood. (a) Case definitions with no qualifying level of parasitaemia; (b) parasitaemia cut-off level ( $d$ ) $=2000$ parasites $/ u \mathrm{~L}$; (c) $d=5000$ parasites $/ u \mathrm{~L}$; (d) $d=10000$ parasites $/ u \mathrm{~L}$; (e) $d=20000$ parasites $/ \mu \mathrm{L}$. The sensitivity is the ratio of the number of cases correctly identified to the total number of cases, and the specificity is the ratio of the number of negative subjects correctly identified to the total number of negatives (BARKER \& HALL, 1991). A CRP cul-orf level of infinity would correspond to case definitions including fever only, ignoring the level of CRP.

tions in afebrile and nocturnal fever patients. Many of these patients with raised CRP were therefore likely to be malaria cases, some of them with intermittent fevers. If the CRP was predominantly a consequence of other conditions there would be no relationship with parasite density.

Although the currently febrile patients had the highest CRP levels, there was negligible correlation between parasite density on presentation and CRP level in this group. Among febrile patients raised CRP is not associated with malaria, since CRP does not discriminate malaria from other febrile or inflammatory conditions (ERIKSSON et al. 1989). However in the febrile patients the highest CRP levels were found one day after presentation, and this again supports the view that CRP can be used as a marker of recent fever. This observation is similar to that of GILLESPIE et al. (1991), who found a CRP peak on the second day after quinine treatment in hospital patients with uncomplicated acute $P$. falciparum malaria. The high correlation between temperature on presentation and CRP levels one day later further supports this interpretation. High CRP levels, associated with high parasitaemia, might therefore be a way of identifying recently febrile malaria cases who do not present with a measurable fever.

An alternative to using fever to define possible malaria episodes is therefore to include all cases with either fever or raised CRP levels or both. In the rather selected group of patients which we have studied, we estimated the sensitivity of fever alone as a diagnostic criterion for malaria at about $60 \%$. This sensitivity can be improved if afebrile patients with raised CRP are included (Fig. 3). The problem with such a CRP dependent case definition is the relatively low specificity. However, the specificity can be improved by requiring the malaria cases to have a high parasite density. We observed that if cases are required to have either raised CRP levels and/or a fever together with a parasite density in the peripheral blood of at least $5000 / \mu \mathrm{L}$, therc is little loss in sensitivity and the specificity is well over $80 \%$.

In community studies subjective and objective measures of malarial morbidity are not always closely correlated (TEUSCHER, 1992). A CRP dependent case definition would be useful in field studies concerned primarily with the underlying biological processes of the disease, since it is likely that only a small proportion of inflammatory responses caused by malaria parasites is detectable either as measurable fever or by fever recall. Such a definition is less appropriate in field studies which are focused on perceived illness at the community level, when identification of episodes by fever recall can be used (TEUSCHER, 1992).

A CRP dependent case definition is less useful to the clinician since in clinical diagnosis and health facilityhased studies a sensitive case definition can more easily be achieved by limiting the exclusions to only those patients with clear alternative diagnoses.

In contrast to our findings with CRP, we judge that haptoglobin is not a useful episode marker in an endemic area. Haptoglobin levels on presentation were highly negatively correlated with parasite density. Haptoglobin levels also showed more serial correlation during convalescence than did parasitaemias. These initial results suggested that ahaptoglobinaemia might indeed be a good indicator of recent hyperparasitaemia, and hence of malaria episodes. However, the magnitude of the correlation between initial parasitaemia and haptoglobin level one day after presentation was less than the serial correlation in the parasitaemias themselves. In contrast to the results for axillary temperatures, parasite density in these patients at any given time itself appeared to be a reasonable indicator of parasite density a day previously (despite the chloroquine treatment). At the same time, the high serial correlation in haptoglobin levels probably reflected the influence of factors other than parasitaemia. For instance, phenotypes with different immunological reactivities exist due to genetic polymorphism (BOREHAM et al., 1981) and this will lead to within-subject correlations in the results of the immunoprecipitation assay.

Hypohaptoglobinaemia thus appears no better a marker of recent hyperparasitaemia than is the current level of parasites and therefore haptoglobin levels do not appear helpful in improving the specificity of malaria case definitions. Since the correlation between parasitaemia and haptoglobin is the same in febrile, presumed malaria patients, and in afebrile predominantly non-malaria patients, low haptoglobin levels are not themselves markers of clinical malaria episodes and therefore cannot be used to improve the sensitivity either. Our results are nevertheless consistent with those of TRAPE \& FRI- 
BOURG-BLANC (1988), who found a significant correlation between ahaptoglobinemia prevalence and parasite density. Low haptoglobin levels generally indicate parasitaemia, rather than malaria morbidity. Haptoglobin levels can therefore be useful in the community assessment of the impact of interventions on the burden of malaria parasitaemia (SISAY et al., 1992; TRAPE et al., 1985).

\section{Acknowledgements}

Our sincere thanks go to the villagers of Namawala and their leaders for their continuous co-operation. We are most grateful to Professor Wen L. Kilama, Director General of the National Institute of Medical Research, Tanzania, for the support of the Kilombero Malaria Project. We also wish to thank the field and laboratory workers of the Ifakara Centre for their excellent collaboration. Discussions with Joanna Armstrong-Schellenberg and Richard Hayes of the London School of Hygiene and Iropical Medicine were also very helpful. We gratefully acknowledge the financial support by the Swiss Directorate for Technical Cooperation and Humanitarian Aid, the Dutch government and the World Health Organization Immunology Research and Training Centre, Geneva. One of us (N.H.) was supported by the Rudolf Geigy Stiftung zu Gunsten des Schweizerischen Tropeninstituts. Research clearance was granted by the Tanzanian Commission for Science and Technology (UTAFITI), as per reference no. NSR/RCA 90.

\section{References}

Barker, D. J. P. \& Hall, A. J. (1991). Practical Epidemiology, 4th edition. Edinburgh: Churchill Livingstone, pp. 16-17.

Boreham, P. F. L., Lenahan, J. K., Port, G. R. \& McGregor, I. A. (1981). Haptoglobin polymorphism and its relationship to malaria infections in The Gambia. Transactions of the Royal Society of Tropical Medicine and Hygiene, 75, 193-200.

Chagnon, A., Yao, N., Carli, P., Paris, J. F., Marlier, S., Pierre, C. \& Bassière, H. (1992). La proteine C-réactive dans l'acces palustre. La Presse Médicale, 21, 217-218.

Chiewsilp, D., Chiewsilp, P., Boonpucknavig, S. \& Rugsakul, T. (1988). Elevation of betaz-microglobin in malaria. Transactions of the Royal Society of Tropical Medicine and Hygiene, 82, 688-689.

Eriksson, B., Hellgren, U. \& Rombo, L. (1989). Changes in erythrocyte sedimentation rate, $\mathrm{C}$-reactive protein and hematological parameters in patients with acute malaria. Fournal of Infectious Diseases, 21, 435-441.

Fleck, A. \& Myers, M. A. (1985). Diagnostic and prognostic significance of the acute-phase proteins. In: The Acute-Phase Response to Injury and Infection, Gordon, A. H. \& Koj, A. (editors) Amsterdam: Elsevier, pp. 249-271.

Gilles, H. M. (1988). The differential diagnosis of malaria. In: Malaria, vol. 1, Wernsdorfer, W. H. \& McGregor, I. (edi(ors). Edinburgh: Churchill Livingstone, pp. 769-779.

Gillespic, S. H., Dow, C., Raynes, J. G., Behrens, R. H., Chiodini, P. L. \& McAdam, K. P. W. J. (1991). Measurement of acute phase proteins for assessing severity of Plasmodium falciparum malaria. Fournal of Clinical Pathology, 44, 228-231.

Govardhini, P., Manoharan, A., Subramanian, S., Mohapatra, S. S. S., Jambulingam, P. \& Das, P. K. (1991). Symptomatic diagnosis of Plasmodium falciparum malaria in field conditions. Indian Fournal of Malariology, 28, 55-62.

Graninger, W. Thalhammer, F., Hollenstein, U., Zotter, G. M. \& Kremsner, P. G. (1992). Serum protein concentrations in Plasmodium falciparum malaria. Acta Tropica, 52, 121-128.

Greenwood, B. M. (1987). Asymptomatic malaria infectionsdo they matter? Parasitology Today, 3, 206-214.

Kilombero Malaria Project (1992). The level of anti-sporozoite antibodies in a highly endemic malaria area and its relationship with exposure to mosquitoes. Transactions of the Royal Society of Tropical Medicine and Hygiene, 86, 499-504.

Kwiatkowski, D. (1991). Cytokines and anti-disease immunity to malaria. Research in Immunology, 142, 707-712.

Kwiatkowski, D. \& Greenwood, B. M. (1989). Why is malaria fever periodic? A hypothesis. Parasitology Today, 5, 264-266.

Kwiatkowski, D., Cannon, J. G., Manogue, K. R., Cerami, A., Dinarello, C. A. \& Greenwood, B. M. (1989). Tumor necrosis factor production and its association with schizont rupture. Clinical and Experimental Immunology, 77, 361-366.
Lyimo, E. O., Msuya, F. H. M., Rwegoshora, R. T., Nicholson, E. A., Munzava, A. E. P., Lines, J. D. \& Curtis, C. F. (1991). Trial of pyrethroid impregnated bednets in an area of Tanzania holoendemic for malaria, Part 3. Effects on the prevalence of malaria parasitaemia and fever. Acta Tropica, 49 $157-163$.

Menon, A., Snow, R. W., Byass, P., Greenwood, B. M. Hayes, R. J. \& N'Jie, A. B. H. N. (1990). Sustained protection against mortality and morbidity from malaria in rural Gambian children by chemoprophylaxis given by village health workers. Transactions of the Royal Society of Tropical Medicine and Hygiene, 84, 768-772.

Mnzava, A. E. P., Rwegoshora, R. T., Tanner, M., Msuya, F. H., Curtins, C. F. \& Irare, S. G. (1993). The effects of house spraying with DDT or lambdacyhalothrin against Anopheles arabiensis on measures of malarial morbidity in children in Tanzania. Acta Tropica, 54, 141-151.

M.O.H. (1983). Guidelines for the Implementation of the PHC Programme in Tanzania. Dar es Salaam: Ministry of Health.

Naik, P. \& Voller, A. (1984). Serum C-reactive protein levels and falciparum malaria. Transactions of the Royal Society of Tropical Medicine and Hygiene, 78, 812-813.

Ree, G. H. (1971). Serum C-reactive protein in Gambian Africans with special reference to $P$. falciparum malaria. Transactions of the Royal Society of Tropical Medicine and Hygiene, 65, 574-580.

Rougemont, A., Bouvier, M., Perrin, L., Yerly, S., Brenner, E., Srivastava, I., Dumbo, O., Soula, G., Tamoura, B. Dolo, A., Kodio, B. \& Ranque, P. (1988). Hypohaptoglobinaemia as an epidemiological and clinical indicator for malaria. Lancet, ii, 709-712.

Shute, G. T. (1988). The microscopic diagnosis of malaria. In: Malaria, vol. 1, Wernsdorfer, W. H. \& McGregor, I. (editors). Edinburgh: Churchill Livingstone, p. 805.

Sisay, F., Byass, P., Snow, R. W., Greenwood, B. M., Perrin, L. H. \& Yerly, S. (1992). Measurement of serum haptoglobin as an indicator of the efficacy of malaria intervention trials Transactions of the Royal Society of Tropical Medicine and Hygiene, 86, 14-16.

Smith, T., Charlwood, J. D., Kihonda, J., Mwankusye, S. Billingsley, P., Meuwissen, J., Lyimo, E., Takken, W., Teuscher, T. \& Tanner, M. (1993). Absence of seasonal variation in malaria parasitaemia in an area of intense seasonal transmission. Acta Tropica, 54, 55-72.

Snow, R. W., Lindsay, S. W., Hayes, R. J. \& Greenwood, B. M. (1988). Permethrin-treated bed nets (mosquito nets) prevent malaria in Gambian children. Transactions of the Royal Society of Tropical Medicine and Hygiene, 82, 838-842.

Stein, C. M. \& Gelfand, M. (1985). The clinical features and laboratory findings in acute Plasmodium falciparum malaria in Harare, Zimbabwe. Central African Journal of Medicine, 9, $166-170$.

Tanner, M., Degremont, A., Savigny, De, D., Freyvogel, T. A., Mayombana, C. \& Tayari, S. (1987). Longitudinal study on the health status of children in a rural Tanzanian community:study area and design. Acta Tropica, 44, 119-136.

Tanner, M., De Savigny, D., Mayombana, C., Hatz, C., Burnier, F., Tayari, S. \& Degremont, A. (1991). Morbidity and mortality at Kilombero, Tanzania, 1982-88. In: Disease and Mortality in Sub-Saharan Africa, Feachem, R. G. \& Jamison, D. T. (editors). Oxford: Oxford University Press, pp. 286305 .

Teuscher, T. (1992). Household-based malaria control in a highly endemic area of Africa (Tanzania): determinants of transmission and disease and indicators for monitoring-Kilombero malaria project. Memórias do Instituto Oswaldo Cruz, 87, supplement 3, 121-130.

Trape, J. F. \& Fribourg-Blanc, A. (1988). Ahaproglobinemia in African populations and its relation to malaria endemicity. American foumal of Epidemiology, 127, 1282-1288.

Trape, J. F., Fribourg-Blanc, A., Bosseno, M. F., Lallemant, M., Engler, R. \& Mouchet, J. (1985). Malaria, cause of ahaptoglobinaemia in Africans. Transactions of the Royal Society of Tropical Medicine and Hygiene, 79, 430-4334.

Received 15 April 1993; revised 2 fune 1993; accepted for publication 2 fune 1993 